\title{
IDENTIDAD PROFESIONAL DE LOS ADMINISTRADORES DE LA CALIDAD EN UNIVERSIDADES CHILENAS: ENTRE LA INVISIBILIZACIÓN Y LA BUROCRATIZACIÓN ${ }^{1}$
}

\author{
Judith Scharager ${ }^{2}$, \\ Paulina Rodrígue $z^{3}$
}

\begin{abstract}
RESUMEN
En las últimas décadas, las universidades han enfrentado crecientes demandas administrativas que han implicado la incorporación de profesionales para la gestión de procesos académicos. Este artículo presenta los resultados de un estudio que explora las percepciones que los profesionales del aseguramiento de la calidad tienen acerca de su propia identidad ocupacional. Para ello, se realizó un estudio cualitativo a partir de entrevistas a integrantes de unidades de aseguramiento de la calidad de universidades chilenas. En los resultados se muestra el proceso de conformación de estas unidades y la manera en que ello contribuye a la configuración de un rol e identidad profesional. Posteriormente, se analiza cómo este grupo percibe su identidad profesional, la cual se caracterizaría por falta de reconocimiento y formas de invisibilización y exclusión en el espacio universitario. Asimismo, se describen las negociaciones que estos profesionales realizan para poder ajustar su identidad al ambiente académico y a las demandas del sistema de acreditación. El artículo concluye discutiendo la necesidad de una mayor integración de las labores académicas y administrativas en las universidades.
\end{abstract}

Conceptos clave: aseguramiento de la calidad, identidad profesional, educación superior.

\section{PROFESSIONAL IDENTITY OF QUALITY MANAGERS IN CHILEAN UNIVERSITIES: BETWEEN INVISIBILITY AND BUREAUCRATIZATION}

\section{ABSTRACT}

In recent decades, universities have faced increasing managerial demands, which has resulted in the incorporation of professionals to manage academic processes. This article presents the results of a study that explores quality assurance professionals' perceptions about their own occupational identity. To do this, a qualitative study was conducted based on interviews with

1 Este estudio es parte de una investigación llevada a cabo para una tesis doctoral en la Universidad de Leiden, titulada "Nuevos actores en una escena antigua: la profesionalización de la gestión de la calidad académica en Chile entre 1990 y 2015" por Judith Scharager, una de las autoras de este artículo.

2 Pontificia Universidad Católica de Chile, Santiago, Chile. Contacto: jscharag@uc.cl

3 Institute of Education, University College, UK. Contacto: paulina.rodriguezanaiz@gmail.com 
members of quality assurance units at Chilean universities. The results show the process of how quality assurance units form and, in turn, how said process shapes the professional role and identity. This is followed by an analysis of this professional group's perception of its professional identity, which is characterized by a lack of recognition and forms of invisibility and exclusion in the university space. The article describes the negotiations that these professionals conduct, in order to adjust their identity to the academic environment and to the accreditation demands. The article concludes by discussing the need for greater integration between academic and administrative work in universities.

Key concepts: higher education, professional identity, quality assurance.

\section{Introducción}

En las últimas décadas, el sistema de educación superior en Chile ha evidenciado un significativo aumento de la cobertura (Servicio Nacional de Información de la Educación Superior, SIES, 2010; 2014). Sin embargo, este crecimiento no necesariamente fue aparejado de un equivalente aumento en la calidad de la oferta educativa. La diversidad de instituciones ha hecho visible importantes diferencias en materia de calidad de los procesos formativos. Esto hizo patente la necesidad de incorporar mecanismos y procedimientos que velasen por la calidad de la formación impartida lo cual, sumado a la influencia de las tendencias internacionales, han constituido factores que han promovido el despliegue de comportamientos "de mercado" en las universidades, con el fin de competir por recursos y ser sustentables en un medio cada vez más competitivo.

Brunner (2017) llama capitalismo académico a las formas en que las economías neoliberales son incorporadas por las instituciones de educación superior como parte de procesos más amplios de racionalización de la modernidad capitalista. Brunner, GangaContreras y Rodríguez-Ponce (2018) se refieren específicamente al caso chileno como "capitalismo académico de mercado". De acuerdo con los autores, debido a la alta privatización de la educación superior en Chile, los mercados juegan un rol en la coordinación del orden interno de las universidades. De este modo, las universidades adoptarían comportamientos gerencialistas y articularían su gobernanza en función de criterios como la eficiencia y la efectividad. 
Es decir, como respuesta a estas condiciones y para hacer frente a las demandas del entorno, las universidades han reaccionado ajustando sus estilos de gestión y sus modelos de financiamiento. Esta permeabilidad ha tenido como consecuencia la introducción de enfoques de administración propios de la empresa privada. Con esta finalidad, las universidades han adoptado estrategias de administración que se orientan a la consecución de metas de desempeño y de productividad, las que requieren ser gestionadas con capacidades técnicas que sobrepasan las competencias académicas.

Como consecuencia de estas adecuaciones, el sistema de educación superior chileno ha experimentado un proceso de modernización y profesionalización de los mecanismos de administración académica. En las universidades se han instalado modelos de gestión derivados de una lógica empresarial influenciados por el enfoque de la nueva gestión pública y las exigencias de un entorno competitivo. Estas condiciones han impulsado importantes transformaciones organizacionales, debido a la incorporación de nuevos ámbitos de acción que requieren de cierto nivel de especialización para hacerse cargo de la creciente demanda de tareas administrativas. En este contexto, se han integrado nuevos profesionales con un perfil orientado a la administración académica, en cargos no siempre bien definidos. De este modo, la dimensión de gestión universitaria ha adquirido un lugar central en las universidades contemporáneas.

La profesionalización de la gestión en las universidades es un fenómeno que ha sido documentado por numerosos autores (Brunner y Pedraja-Rejas, 2017; Cáceres, 2007; Deem, Hillyard \& Ree, 2007; Fernández, 2009; Watermeyer, 2015; entre otros). La literatura especializada es abundante y revela una predominancia de estudios que sistematizan la percepción de los académicos acerca de la administración universitaria (Degn, 2015; Henkel, 2000; 2005). Sin embargo, la evidencia que caracteriza las perspectivas de los propios administradores acerca de su inserción en las universidades es muy escasa y se concentra en países anglosajones (Deem et al., 2007; Lewis, 2014; Whitchurch, 2006; 2007; 2008; 2013; Winter, 2009). Muchos de estos estudios sugieren que se han disuelto los 
límites entre las áreas funcionales, profesionales y académicas, configurándose un "tercer espacio" (Whitchurch, 2006), el cual es ocupado principalmente por profesionales con un perfil técnico y, excepcionalmente, académicos con talento para la gestión.

Al igual que en países anglosajones, muchas universidades chilenas han cambiado la tradicional estructura de apoyo administrativo por una de gestión profesionalizada (Scharager, 2017; 2018). Este cambio se aplica en distintos ámbitos de la administración académica y, en los últimos años, en particular en el ámbito del aseguramiento de la calidad, producto de la instalación de los sistemas de evaluación y acreditación institucional y de programas de estudio. Históricamente, la actividad administrativa se desarrollaba como un servicio de apoyo para el cuerpo académico, ofrecido por personal sin preparación especializada. Es decir, había un límite claro entre lo que se consideraba "de la administración" y "de la actividad académica". A medida que aumentó la carga administrativa, se fueron introduciendo sistemas que han requerido una progresiva profesionalización de la gestión y una dotación cada vez más numerosa de administradores con estudios superiores. Un indicador del aumento de estos profesionales se puede observar en las bases de datos de contratación de personal no académico con título profesional de las universidades del Consejo de Rectores de las Universidades Chilenas, $\mathrm{CRUCH}^{4}$. En estos registros se observan incrementos de hasta 182\% en esta dotación en algunas universidades (CRUCH, 2014), llegando casi a duplicar la dotación de personal académico de jornada completa.

El desarrollo de estos espacios de gestión con fines de apoyo técnico a las actividades académicas dentro de las instituciones ha introducido no solo nuevos actores, sino que también nuevas nociones respecto de una serie de aspectos constitutivos de las universidades, como las formas de financiamiento, el control del quehacer académico y, en particular, la calidad de la educación y cómo se debe gestionar.

4 El Consejo de Rectores de las Universidades Chilenas (CRUCH) es una persona jurídica de derecho público, de administración autónoma, creado por ley el 14 de agosto de 1954, como un organismo de coordinación de la labor universitaria de la nación. Está integrado por los rectores de las 25 universidades públicas y tradicionales del país, creadas antes de 1981 o derivadas de ellas. Más información en consejoderectores.cl 
De este modo, han comenzado a coexistir distintas concepciones y discursos que podrían ser antagónicos al punto de generar tensiones entre una orientación académica y la administrativa. La literatura especializada muestra que los académicos no comparten las mismas visiones en torno a la educación de los "no-académicos" y creen que su integración en las universidades le ha restado poder a los cuerpos colegiados (Biggs, 2001; Harvey, 2004; Newton, 2002; Parri, 2006). Además, la ideología que subyace a las funciones que realizan, vinculadas con la administración y burocratización de procesos, es percibida como contraria a la naturaleza académica de las universidades (Espinosa, 2015; Winter, 2009).

En este contexto, los nuevos profesionales paulatinamente han asumido funciones y tareas que derivan de la complejización de los requerimientos administrativos que debe enfrentar una universidad. Entre las tareas que asumen se pueden mencionar la preparación y apoyo a los procesos de toma de decisiones, el manejo de las relaciones internacionales, el desarrollo de programas curriculares específicos, la gestión de centros de estudios y proyectos de investigación y la administración de estrategias para asegurar la calidad y procesos de acreditación. De estas acciones, la acreditación de instituciones y programas adquiere una especial relevancia en Chile, porque el sistema de aseguramiento de calidad está regulado por ley desde el año 2006. Bajo la normativa vigente, las universidades se abocan al cumplimiento de las demandas de transparencia y rendición de cuentas, las cuales se han convertido en condición para la obtención de recursos financieros. Por ello, los procedimientos de aseguramiento de la calidad no solo son una forma de medición, sino que se han convertido en una dimensión que está integrada en las universidades y que estructura particulares modos de funcionamiento dentro de estas (Barnetson \& Cutright, 2000; Engebretsen, Heggen \& Eilertsen, 2012; Filippakou, 2001; 2017; Morley; 2003). Los procesos de aseguramiento de la calidad traducen ciertas racionalidades en modos particulares de gobernanza y de profesionalidad. Morley (2003), por ejemplo, sugiere que los sistemas de aseguramiento de calidad han implicado una transferencia de tecnologías desde el sector privado al público. Estas tecnologías valorizan el rendimiento y la eficiencia en las instituciones de educación superior. Geoffroy Pitta (2013) 
sugiere que el sistema de aseguramiento de la calidad está articulado sobre la capacidad de autorregulación y el cumplimiento de objetivos organizacionales autoimpuestos por las propias instituciones, lo que supone que estas ajustan sus acciones a dichos objetivos y también a los estándares fijados externamente. La progresiva internalización de las funciones de auditoría junto con las crecientes demandas de autoinspección organizacional por parte de las universidades, ha llevado a la incorporación de "profesionales de aseguramiento de la calidad" para hacerse cargo de esta ineludible tarea.

Las demandas de calidad se vieron intensificadas a partir de la puesta en marcha de la Ley No 21.091 Sobre Educación Superior, promulgada en Chile en mayo de 2018. En ella no solo se establece la obligatoriedad de los procesos de acreditación institucional y de las carreras de Pedagogía, Medicina y Odontología, sino que además se establece como una nueva dimensión a evaluar la existencia de un mecanismo de aseguramiento interno de la calidad. Esta condición, sin duda, impactará en el incremento de contratación de profesionales que conformen dicho mecanismo, con el fin de cumplir con el requisito ineludible estatuido como condición para obtener la acreditación institucional. En este marco, crece el abanico de requerimientos de competencias técnicas en materia de gestión, que ya fue impulsado por la Ley No 20.129 del año 2006, y se fortalece la necesidad de contar con unidades de aseguramiento de calidad y profesionales a cargo dentro de las universidades.

Estas unidades, establecidas a partir de los años noventa como Direcciones, Oficinas o incluso como Vicerrectorías de Aseguramiento de la Calidad (González et al., 2007; Rivera, Astudillo y Fernández, 2009), incorporaron profesionales abocados a un nuevo rol: la administración de procesos de acreditación. Conforme los sistemas de aseguramiento de la calidad han cobrado más relevancia, las funciones que desarrollan se han ido tecnificando y ampliado progresivamente. Lemaitre (2015), en relación con la profesionalización del aseguramiento de la calidad en Chile, la describe como "el desarrollo de un conjunto reconocido de especialistas, un cuerpo organizado de conocimientos, el establecimiento de procesos de capacitación sistemáticos y la instauración de asociaciones profesionales de quienes 
se dedican a esta actividad" (p. 299). La autora señala que es posible encontrar todos estos elementos en el caso chileno.

En este marco, surgen interrogantes respecto del proceso de integración de los profesionales del aseguramiento de la calidad en las universidades y su construcción identitaria en el marco de la modernización de los sistemas de educación superior, bajo las regulaciones de la ley vigente. Si bien es un tema explorado en la literatura internacional, en Chile la evidencia se ha centrado en el estudio de la profesionalización de la academia (por ejemplo, Bernasconi, 2008; Berríos, 2015), en tanto la incorporación de profesionales que realizan labores de gestión en las universidades ha recibido escasa atención. En este contexto, este estudio tiene por objeto explorar las percepciones que los profesionales del aseguramiento de la calidad en universidades chilenas tienen acerca de su propia identidad profesional en el medio académico.

En la primera parte del artículo se describe la concepción de identidad que aborda este estudio y la literatura, mayormente anglosajona, que describe la experiencia laboral del nuevo grupo de profesionales dedicados a la gestión académica en las universidades. A continuación, se explicita la metodología de carácter cualitativa utilizada en el estudio. La tercera sección del artículo muestra los resultados donde se destacan dos dinámicas: la primera describe el proceso de instalación de las unidades de aseguramiento de la calidad en las universidades de los participantes del estudio y la manera en que ello configuró un rol profesional; la segunda da cuenta de cómo los profesionales son invisibilizados en el contexto académico y la manera en que recurren a la burocratización de procesos como estrategia para negociar un espacio identitario. Finalmente, en la discusión se analizan los principales hallazgos del estudio a la luz de la literatura.

\section{La identidad profesional en el proceso de profesionalización de la gestión académica}

Desde la tradición del constructivismo social, la identidad ha sido comprendida como una construcción derivada de un proceso reflexivo en torno a las experiencias vividas y a la participación en 
discursos sociales. Es decir, es concebida como un proceso de diálogo y confrontación entre lo interno y externo; una interacción dinámica entre el "encasillamiento" de la identidad atribuida por otros y el intento de proyectar una identidad hacia el exterior. De esta forma, esta requiere ser comprendida como una interacción coconstruida entre las personas y las estructuras sociales. Por ejemplo, Dubar (1992) menciona dos dinámicas identitarias. La transacción objetiva o relacional ocurre entre el individuo y los otros significativos, con el fin de acomodar o equilibrar la identidad para sí y la identidad para el otro; es una operación de reconocimiento y legitimidad de sus pretensiones, teniendo en cuenta los objetivos y los medios ofrecidos. Por otro lado, la transacción subjetiva o biográfica implica esfuerzos para salvaguardar parte de sus identificaciones anteriores e identidades atribuidas y el deseo de construir nuevas identidades; es decir, está en el proyectar futuros posibles en continuidad o ruptura con un pasado reconstruido (Dubar, 1992).

Si bien las identidades ocupacionales en las universidades se han definido tradicionalmente a través de ámbitos estructurados como las disciplinas; el desdibujamiento de los límites institucionales y las exigencias políticas de la educación superior han promovido la emergencia de un grupo cada vez más multiprofesional y diverso en la medida en que el trabajo se orienta por proyectos que cruzan fronteras funcionales y disciplinares. Los cambios, entonces, no solo se producen a nivel organizacional sino también a nivel identitario individual.

Allen-Collinson (2006), estudiando las identidades ocupacionales de administradores de investigación en universidades de Inglaterra, observó que sus concepciones identitarias estaban fuertemente influenciadas por los colegas administrativos y por los académicos. En conjunto, constituían los "otros" significativos para este grupo profesional. De acuerdo con la autora, la naturaleza jerárquica de las universidades, con académicos ubicados muy por encima de los administradores de investigación en la estructura de poder, permite a estos imponer su "categoría" sobre los otros grupos ocupacionales dentro de la universidad. De esta forma, los administradores de investigación en las universidades han sido definidos como "otros" y como "no-académicos". Esta clasificación 
fue vivenciada como problemática por muchos entrevistados, quienes expresaron gran frustración por lo que percibían como "una identidad por defecto" de "no-académico", la que fue considerada como denigrante y como medio para negar sus conocimientos y sus habilidades. Los hallazgos de McInnis (1998), estudiando a administradores académicos de universidades australianas son muy convergentes. Por ejemplo, señala que este grupo profesional no estaría conforme con su identidad de "no académicos" y sentiría escaso reconocimiento por la labor de gestión que realizan, así como por su experticia y capacidades.

Otra característica relevada en la literatura es la invisibilización de sus funciones. Szekeres $(2004,2011)$ al igual que Allen-Collinson (2006), señala que la mayoría de los administradores considera que tanto ellos como sus trabajos son "tomados por sentado" o en gran medida invisibilizados. Al respecto, Whitchurch (2007) sostiene que hay poco reconocimiento de la existencia de un grupo ocupacional dedicado a la gestión, más allá de los mismos profesionales que lo componen. Además, dado que académicos y estudiantes son concebidos como quienes conforman el núcleo de las universidades; por oposición, el cuerpo profesional es visto como la periferia. Para Allen-Collinson (2006), los administradores se perciben a sí mismos como un miembro marginal de la corriente principal de la comunidad académica.

Otro aspecto señalado en la literatura como característico del trabajo de los administradores es la falta de definición de sus labores, siendo difícil situar estos roles dentro de fronteras prescritas (Whitchurch, 2004). Este cruce de límites y la naturaleza ambigua del rol parecen congruentes con el concepto de liminalidad, desarrollado en la década de los años sesenta por Van Gennep (2008) en el contexto de la antropología. Inicialmente aludía a "ritos de pasaje" que permitían la transición a una nueva identidad. El concepto hace referencia a un estado ambiguo, indeterminado, en el que una persona se encuentra atrapada en un espacio intermedio de transición identitaria. Es decir, cuando una persona está en medio de dos construcciones de identidad: no es una cosa ni la otra, está en un espacio transicional. Para contrarrestar esta identidad indefinida, 
los administradores adoptarían diversas estrategias de modo de compensar su indefinición, hacer notar su labor dentro de las instituciones y manejar la ansiedad que les generan las condiciones de incertidumbre o de ambivalencia.

De acuerdo con Brown y Starkey (2000), en las organizaciones hay ciertas prácticas que pretenden convertir las ambigüedades en situaciones más tolerables; las que se pueden expresar a través de artefactos lingüísticos y visuales, como historias, mitos, chistes y chismes, que simbólicamente operan como un proceso que ayuda a enfrentar estas situaciones poco definidas o percibidas como amenazantes. En esta misma línea, Wastell (1996) muestra cómo esta disociación se resuelve empleando métodos estructurados de manera ritual para defenderse de la ansiedad que genera esta situación ambivalente. Ese autor argumenta que, en situaciones poco definidas, las personas despliegan manifestaciones de control de la ansiedad social dentro de las organizaciones, mediante los papeleos excesivos o la sobrevaloración y ritualización de las técnicas y procesos administrativos que le otorgan una sensación de seguridad. De esta forma, acorde con los planteamientos de Wastell (1996), las técnicas, formularios y procesos administrativos pueden ser vistos como objetos que ayudan a transitar entre un estado ambiguo de construcción de identidad y uno más maduro y asentado. Sin embargo, en la práctica se puede desarrollar una dependencia poco saludable con el espacio transicional y con los procedimientos administrativos al punto que estos se convierten en una técnica utilizada como un fin y no como un medio.

A la falta de delimitación en el rol se suma el hecho de que los términos "administración" y "gestión" no solo carecen de precisión, sino que son descalificados en el contexto académico. Estos serían asociados con una burocracia indeseada, con la erosión de la autonomía académica y con excesivos requerimientos de rendición de cuentas (Whitchurch, 2008). Según McInnis (1998), el hecho de instalar nuevas funciones administrativas habría traído a las instituciones valores asociados al individualismo y al mercado, donde las preocupaciones típicas serían la eficiencia, efectividad, productividad y rendimiento. Todas ellas, dificultarían 
la relación de los administradores con los académicos, dado que serían percibidos por estos como mensajeros de la burocracia (Allen-Collinson, 2006). También se ha señalado que en los académicos primaría un anhelo nostálgico asociado a la pérdida de la libertad académica y autonomía en el trabajo (Henkel, 2007; Ylijoki, 2005). Esto porque la actividad académica se fundamentaría principalmente en recompensas ideológicas y abstractas, tales como el valor de la disciplina, la curiosidad intelectual, la posibilidad de insertarse en una comunidad de práctica y la autorregulación. Por ello, los planteamientos gerencialistas tienden a percibirse como contrarios a los ideales defendidos por los académicos (Winter, 2009). Los procesos burocráticos aparecerían corporeizados en los administradores, quienes deben lidiar con la frustración de los académicos al ver constreñidos sus valores en favor de una identidad gobernada por una racionalidad económica y administrativa.

En consecuencia, los administradores y académicos han ido conformando no solo grupos con identidades diferenciadas, sino también con valores y directrices muy divergentes (Seyd, 2000). Debido a que no hay historia ocupacional previa de estos cargos, es esperable que la influencia externa permee más fuertemente en este nuevo grupo profesional moldeado por las nuevas demandas de gestión universitarias. En el contexto actual, se requiere generar evidencia que permita comprender el proceso de inserción de este nuevo grupo en las universidades. Este artículo presenta los resultados de un estudio que exploró la profesionalización de la gestión del aseguramiento de la calidad universitaria en Chile. Particularmente, se analizaron los procesos de configuración de la identidad profesional de este nuevo rol en las universidades. Se exploran las tensiones y desafíos que enfrentan, así como las estrategias que utilizan para negociar un espacio identitario.

\section{Metodología del estudio}

El estudio forma parte de una tesis doctoral (Scharager, 2017) que consideró dos fases, siguiendo un diseño mixto y secuencial. En la primera, se encuestó a integrantes de las unidades de aseguramiento de la calidad y acreditación de 48 universidades chilenas. La encuesta indagó 
en su trayectoria profesional, perfil sociodemográfico, descripción del cargo y funciones. La segunda fase consistió en un estudio cualitativo que tuvo como objeto explorar las percepciones que los profesionales del aseguramiento de la calidad tienen acerca de la calidad y de su propia identidad profesional. Para ello, se realizó un muestreo intencionado entre quienes participaron de la primera fase y que habían consentido ser entrevistados. Se privilegió contar con un amplio espectro en cuanto a edad, género, formación inicial y universidad en la cual trabajaba como administrador de la calidad. Formaron parte del estudio 20 profesionales de unidades de aseguramiento de la calidad de 19 universidades chilenas; nueve pertenecientes al CRUCH y 10 a universidades privadas no pertenecientes al CRUCH. Todos los participantes al momento de la entrevista tenían funciones de gestión del aseguramiento de la calidad en sus universidades. Algunos de ellos lideraban las unidades institucionales de aseguramiento de la calidad, mientras que otros conducían los procesos de acreditación a nivel departamental o de facultad.

Para orientar las entrevistas, se elaboró una pauta semiestructurada que contenía cuatro secciones. Esta pauta fue probada inicialmente con una profesional que desempeñaba funciones semejantes a la de los participantes. Esta entrevista no fue incluida en la muestra final. Luego de la retroalimentación, se hicieron los ajustes necesarios. La pauta de entrevista abordó aspectos relacionados con la trayectoria laboral, profesionalización de la gestión académica, concepciones de calidad y construcción identitaria y del rol ocupacional. En este artículo se presenta uno de los temas estudiados, referido a la identidad profesional y a la configuración de su rol dentro de las universidades chilenas.

Las entrevistas tuvieron una duración de 60 minutos aproximadamente y fueron conducidas por las autoras de este artículo. Las entrevistas fueron grabadas y transcritas posteriormente. Los participantes suscribieron un consentimiento informado donde se detallaron los objetivos del estudio y el manejo de la información. Todos los nombres de los participantes han sido sustituidos por un pseudónimo y se eliminaron todas las referencias de sus instituciones. 
El análisis del material contempló, en primer lugar, la transcripción de cada una de las entrevistas y, posteriormente, su procesamiento utilizando los pasos del análisis temático propuesto por Braun y Clarke (2006). Este método analítico permite identificar patrones temáticos dentro de los datos. La primera etapa consideró la producción de códigos iniciales guiados por la literatura en el área. Luego, los códigos fueron agrupados para formar categorías más amplias o temas, los cuales fueron agrupados en una matriz de datos. Los temas fueron revisados en función de la consistencia interna con el set de datos y a nivel de participante. Se utilizó el software Nvivo 10 para todo el proceso de análisis de las entrevistas.

\section{Resultados}

A continuación, se presentan los principales resultados obtenidos, los cuales son ilustrados con las citas más representativas señaladas por los profesionales. Las citas sustentan las interpretaciones elaboradas y ejemplifican los rasgos más distintivos de los temas construidos. Los resultados descritos a continuación fueron organizados en dos apartados. El primero describe el proceso de instalación de las unidades de aseguramiento de la calidad en las universidades y la manera en que ello contribuyó a la configuración de un rol profesional. El segundo apartado muestra cómo los entrevistados son invisibilizados en el contexto académico y las negociaciones que realizan para enfrentar esta invisibilización.

3.1. Marcando territorio: la conformación de las unidades de aseguramiento de la calidad en las universidades

Las universidades contemporáneas llevan a cabo sus funciones en un marco de educación masiva y de mercado que les plantea exigencias de rendición de cuentas públicas y de alta competitividad. Estas condiciones han propiciado la adopción de medidas de eficiencia y eficacia que emulan al sector empresarial, aplicando estrategias gerencialistas, como se describió anteriormente. Whitchurch (2006) señala que las prácticas de administración académica se han instalado sobre las estructuras y culturas tradicionales de las universidades, haciendo más permeables las fronteras internas y externas de las 
instituciones. Esto ha facilitado la integración de profesionales con experiencias ajenas a la academia para hacerse cargo de las nuevas tareas de gestión, entre las que destacan las orientadas al aseguramiento de la calidad. Estos profesionales cumplen un rol de articular al interior de la institución en distintos niveles de la universidad y también de relacionarse con entidades del entorno externo, en particular, con los organismos que demandan rendición de cuentas, como es el caso del Ministerio de Educación de Chile y la Comisión Nacional de Acreditación. Es decir, son como bisagras o piezas intermedias, pero sin una función muy delimitada. Debido a la falta de definición del rol y tareas centrales que deben cumplir en la universidad, una de las características distintivas de quienes se desempeñan en estas unidades es la percepción de sí mismos como pioneros:

Todo lo que existe a la fecha en la unidad ha sido creado por quien responde: la misión, funciones, perfil de los cargos en la unidad, políticas de autoevaluación, etc. (Juan, universidad del CRUCH).

Desde el establecimiento de estas oficinas de aseguramiento de calidad, su definición en términos de ámbitos de acción ha sido poco precisa y su organización en la estructura universitaria ha ido perfilándose sobre la marcha. Dado que los integrantes de estas unidades de gestión han sido los primeros en ocupar estos cargos y no hay precedentes de experiencias que orienten el camino a seguir, no solo son pioneros, sino que los potenciales "constructores de cultura" en este nuevo espacio de administración académica. Sin embargo, advierten que la instalación de estas unidades ha sido poco comprendida en el contexto académico. Por ello, describen su experiencia de inserción en las universidades como difícil, al percibir que son visualizados como foráneos en el contexto académico, tal como lo expresa una de las participantes:

Hace ocho años atrás, cuando llegábamos a las unidades nos miraban como “¿Estos extraterrestres a qué vienen?” (María, universidad del CRUCH).

María alude a la idea de "extraterrestres" para graficar cómo fueron considerados inicialmente en la universidad: figuras ajenas a 
la tradicional conformación universitaria. Esto es consistente con la introducción de modos de gestión en el marco de la nueva gestión pública en las universidades. La incorporación de profesionales ajenos a la universidad podría favorecer la apropiación de una perspectiva disociada de la cultura académica y alejada de la misión esencial de las universidades, en especial, en aquellas instituciones donde los cuerpos académicos son más débiles. En el caso particular de la gestión del aseguramiento de la calidad esta disociación se potencia debido a que, por lo general, las funciones administrativas se alojan en una estructura organizacional ad hoc, concebida especialmente y dispuesta cerca de la línea de mando institucional, en posiciones distantes del trabajo académico concreto. Estos dispositivos están más bien en la "retaguardia", de modo que la disposición organizacional de las funciones implícitamente asume como supuesto que la calidad es manejable como un componente independiente o paralelo al quehacer académico:

es una Vicerrectoría independiente de la Vicerrectoría Académica, para separar los procesos de lo que es autoevaluación y acreditación, de los procesos académicos propiamente tales (...). Y la idea es que una Vicerrectoría de Aseguramiento de la Calidad mire los procesos, pero desde otro ámbito, desde afuera, con el fin de ir evaluando cómo se están desarrollando, cómo se están llevando a cabo. Entonces, hace más transparente y hace más eficiente tanto una cosa como la otra, porque no se mezclan... parece razonable que todo lo que tenga que ver con aseguramiento de la calidad no esté metido dentro de la misma unidad que es la que lleva a cabo los procesos académicos (Cecilia, universidad no-CRUCH).

Morley (2003) argumenta que el término "nuevo" en relación con la nueva gestión pública, puede de hecho aludir a formas antiguas de gestión. Sostiene que una versión del taylorismo, como forma "científica" de control de la producción, es ahora aplicada a la labor intelectual. Morley (2003) explica que el aseguramiento de la calidad demanda que la actividad académica sea dividida en pequeñas partes manejables. Así, a través de la fragmentación, la calidad es reducida a piezas, lo cual supone que esta puede ser diseñada y controlada 
a través de prácticas y estructuras organizacionales estables y reproducibles. Por otro lado, la opción de separar la gestión de calidad de la cotidianeidad académica y delegarla a una unidad diferenciada, parece haber reforzado una racionalidad y un discurso impregnado de tecnicismos administrativos más que académicos. Como consecuencia, la mayoría de las unidades de aseguramiento de calidad chilenas organizan su trabajo en torno a tareas abocadas a apoyar prácticas de planificación operativa y estratégica para cumplir con las exigencias externas y mostrar resultados que serán reconocidos en número de años de acreditación. Esto comprende la generación de indicadores y conducción de procesos estandarizados, regidos por manuales y reglamentos, que siguen una lógica de organización del trabajo propia de la empresa. La naturaleza y funciones de la universidad, asociadas a la creación y la transmisión de conocimiento, parecen quedar en un segundo plano. De hecho, como lo expone una de las profesionales entrevistadas cuando se le pide definir su trabajo, desde su perspectiva la universidad es concebida como "otra empresa más":

hay una transferencia entre lo que pasa en las otras empresas con el tema, porque nosotros hemos asumido la norma ISO. Porque la norma ISO dispone de una serie de mecanismos que permiten la autorregulación permanente, es fácil de manejar, es fácil de hacerle las observaciones técnicas, y por lo tanto es adecuado. (...) tenemos un manual de calidad (...) y por lo tanto esperamos que la instalación adecuada de este manual permita instalar en forma permanente mecanismos de autorregulación institucional, de manera de autorregular más allá de la acreditación (Cecilia, universidad no-CRUCH).

La introducción de técnicas propias de la empresa privada intenta, entonces, convertir aspectos tácitos de la educación en procesos manejables, calculables y estandarizables, que puedan ser "fáciles de manejar" como señala la entrevistada. En consecuencia, la labor de estas unidades de gestión y de sus integrantes tiende a concentrarse en asuntos de carácter instrumental y administrativo, priorizando los procedimientos y los sistemas de registro, en respuesta a las demandas externas. Es decir, desde la instalación de estas unidades en las universidades, se ha privilegiado su 
localización distante del trabajo de los académicos, con funciones establecidas para responder a demandas administrativas del medio externo. Por este motivo, los profesionales de estas unidades deben negociar, para recabar datos de productividad institucional, con un ambiente que se opone a realizar acciones percibidas como contrarias al quehacer central de una universidad. En este proceso, realizan constantes esfuerzos por delimitar y marcar un territorio, a través de la apropiación de procedimientos que los distinguen y empoderan. Estas acciones tienen impactos en la identidad profesional que construyen, como se describirá en la siguiente sección.

\subsection{Identidad en disputa: reconocimiento, invisibilización y burocratización}

\section{a. Luchas por reconocimiento}

En el marco de la reciente creación de unidades de aseguramiento de la calidad, los participantes de este estudio mencionaron preocupaciones relativas al reconocimiento de su rol en las universidades. Esta búsqueda de reconocimiento y visibilidad fue común a los administradores entrevistados, particularmente, la necesidad de validarse como interlocutores legítimos y el anhelo de poder funcionar de manera colaborativa y creativa con el mundo académico. De esta forma, los administradores negocian activamente un espacio profesional, que no esté dado por sentado, bajo la mirada y la aceptación del cuerpo académico. Por ejemplo, la siguiente cita ilustra cómo una encargada de una unidad de calidad intenta posicionarse como una experta en un campo, con el fin de negociar un estatus identitario que pueda equiparase al del cuerpo académico:

por lo tanto también hay que ganarse el respeto de los académicos, porque son los principales actores de un proceso de esta naturaleza en la unidad académica. Entonces hay que lograr posicionarse casi como un par, como un par que es experto en un área y que lo viene a acompañar y ayudar en un proceso, y un proceso que inicialmente es algo adicional y le significa más trabajo al académico (Ana, universidad del CRUCH). 
No obstante, la introducción de técnicas de administración propias del gerencialismo ha impuesto nuevos modelos de trabajo que también han impactado la profesionalidad de los académicos. Como señala Ana en la cita anterior, los procesos de aseguramiento de la calidad son percibidos como ajenos y como una carga adicional para el cuerpo académico. Thomas y Davies (2002) advierten que las prácticas derivadas de esta lógica han producido un proceso gradual de desprofesionalización en los académicos, pérdida de la autonomía profesional y transformación de la labor académica en un tipo de commodity, todo ello con profundas implicancias en su identidad profesional. De un modo similar, Anderson (2006) en Australia sostiene que los académicos evalúan negativamente los mecanismos de aseguramiento de la calidad. Si bien permanecían comprometidos con la calidad de la enseñanza, consideraron que los procedimientos de aseguramiento de calidad imponían una excesiva carga de trabajo que fallaba en asegurar la calidad de manera significativa. Estas dinámicas con el cuerpo académico pueden explicar, en parte, las dificultades en la interacción que reportan los administradores de la calidad. De esta forma, las habilidades para lidiar con la resistencia del cuerpo académico se vuelven centrales en el repertorio de estos profesionales. Como ilustra una de las informantes, las habilidades interpersonales son cruciales para poder "convencer" a los académicos acerca de la relevancia de las funciones del aseguramiento de la calidad:

Primero, porque yo creo que la cultura académica es adversa aún a procesos que la sacan de su ethos, que es la academia, enseñar, investigar, gestionar la unidad. Por lo tanto, uno tiene que ser capaz de seducir al director de departamento, al jefe de carrera. Seducir a esa comunidad y a sus líderes para involucrarse en un proceso de esta naturaleza más allá del ritual organizacional. Y ahí uno tiene que ser un poco un coach o un inspirador de ese grupo (Daniela, universidad del CRUCH).

Es decir, los administradores de la calidad buscan reconocimiento a su identidad profesional en un espacio en el cual son percibidos como los portadores de la burocracia y la administración $y$ en donde sus funciones son consideradas alejadas de la academia 
propiamente tal. Por ello, el despliegue de habilidades interpersonales resulta central para construir una identidad positiva y presentarse a sí mismos como pares con conocimientos específicos que pueden aportar a las instituciones.

\section{b. Invisibilidad, periferia e identidad por defecto}

Otro aspecto señalado por los administradores, consistente con la literatura antes planteada, es la invisibilidad al interior de las universidades (Allen-Collinson, 2006; 2009; McInnis, 1998, Szekeres, 2004; Whitchurch, 2006). Por ejemplo, varios de los entrevistados señalaron que son marginados de los espacios de poder e influencia universitaria, los cuales estarían reservados solo para los académicos. Aún más, esta falta de visibilidad social parece extenderse más allá de lo metafórico, resultando en una forma activa de exclusión por parte de los académicos. En la siguiente cita, una de las entrevistadas describe cómo fue posicionada como invisible y no importante al momento de incorporarse en una universidad.

Me dijeron (...) bueno, pero usted se viene, pero tenga en claro que usted acá no existe, usted acá es un NN, toda su experiencia acá no sirve, porque en realidad nosotros somos la Universidad XX y nosotros somos la X universidad de Chile en importancia. Usted para los académicos de esta universidad tampoco existe, no tiene la profesión correspondiente, por lo tanto, tampoco tiene valor la profesión que usted tiene (Violeta, universidad del CRUCH).

La cita anterior ilustra no solo cómo la naturaleza jerárquica de la academia es preservada a través de la exclusión de otros, sino también los discursos que promueven una construcción desde la inferioridad. En este caso, la representación como invisible, "usted acá no existe", es una forma de estigmatización y de denigrar las habilidades, a la vez que permite mantener al otro "en su lugar". Los entrevistados mencionaron otras formas de clasificación negativas al interior de las instituciones, las cuales significaban frustración para algunos de ellos. Por ejemplo, Carolina expresa cómo los productos de su labor no tienen notoriedad y parecieran no colaborar con las tareas centrales que realiza la academia. Es decir, este grupo 
profesional es posicionado como afuera de la corriente principal de la universidad, lo que promueve una "identidad desde la periferia":

Tengo que ser súper clara porque aunque tú a veces hagas quinientas cosas, y no se ven, y no las ven. No voy a hablar del valor, si las valoran o no porque es relativo, pero no se ven. A veces la gestión no se logra ver, no logra ser tan notoria. Por ejemplo, el tema de los académicos es mucho más notorio, gradúan a sus alumnos, sus alumnos tienen empleabilidad específica, tienen puestos de trabajo en específico y tú dices ¿qué vela del entierro tengo yo en eso? y tú puedes decir, ninguna, o toda, depende de cómo yo lo quiera ver (Carolina, universidad del CRUCH).

Un último aspecto señalado, coincidentemente con la literatura, es la definición de su identidad ocupacional en gran medida por lo que no son - "no-académicos"—y, por tanto, como el "otro" (AllenCollinson, 2006). La siguiente cita ilustra cómo, al explicar en qué consiste su rol, los profesionales de la gestión de la calidad se definen por oposición, construyendo "una identidad por defecto" (McInnis, 1998):

Lo voy a responder tal cual como si me hubiera preguntado alguien en la calle: "mira, yo trabajo en temas de gestión en una universidad", a veces pasan, pero hay gente que lo empieza a entender, "ah, o sea, no eres propiamente académico, sino que estás metido en otras áreas" (Miguel, universidad del CRUCH).

c. Negociaciones identitarias: acreditación y burocratización Con el fin de contrarrestar una identidad problemática y la exclusión en la academia, los profesionales tenderían a enfatizar la estandarización de procedimientos y buscarían vincular su trabajo a procesos concretos. Particularmente, la acreditación les permite alcanzar logros tangibles y adquirir visibilidad. De esta forma, la mayoría de los entrevistados se describieron como encargados de acreditación:

O sea, yo soy la encargada de acreditación de la Universidad X. Eso digo, para hacerla corta, digamos. Sí, encargada de acreditación de pregrado de la Universidad X (Violeta, universidad del CRUCH). 
En este proceso de definición identitaria, en un entorno en que el "otro" (académico) tiene su identidad más definida y arraigada a su adscripción disciplinaria, este nuevo actor debe recurrir a estrategias que lo ayuden a reducir la ansiedad propia de las situaciones indefinidas y ambiguas. De esta forma, la definición desde los procesos de acreditación permite empoderar a los administradores a través de una autoridad dada por el cuerpo externo de regulación. Esto se materializa en la escritura de informes y en recolección de datos.

[Yo] era como el escritor fantasma de toda la parte de gestión institucional. Y ahí tuve que hacer toda la pega, diseñar el proceso, levantar las encuestas, generar los focus, hablar, transversalizar los académicos, generar consenso, concluir, emitir informes, hacer [listas de] fortalezas y debilidades... toda el área, porque en el fondo era... todo para la acreditación... (Teresa, universidad del CRUCH).

Sin embargo, el foco en la acreditación y las demandas de información que este conlleva también pueden ser comprendidos como parte de procesos más amplios de requerimientos de performatividad. Ball (2003) discute los efectos de la performatividad en el sector público, la cual describe como una tecnología, una cultura y un modo de regulación que utiliza juicios y comparaciones como medios para gobernar. Sostiene que los sistemas de monitoreo y producción de información, que están al centro de las reformas educacionales, han provocado una inseguridad ontológica en quienes trabajan en estas instituciones. Ball (2003) argumenta que esto ha llevado a presiones constantes por mostrar efectividad, donde el esfuerzo y el tiempo en tareas de primer orden (enseñanza, investigación, etc.) son invertidos en tareas de segundo orden (trabajo en indicadores de performance). Cuando los requerimientos de performatividad cobran supremacía, Ball (2003) sostiene que se corre el riesgo de que auténticas relaciones sociales sean reemplazadas por relaciones de juicio y evaluación de las personas en función de su productividad. Es decir, el énfasis en la performance produce una alienación del yo, a través del desplazamiento de las cualidades individuales. Teresa, en el extracto anterior, se describe como una "escritora fantasma". Además de remarcar una forma de invisibilidad, 
como fue mencionado anteriormente, adopta una identidad profesional construida para ser responsiva a los requerimientos externos y como alguien que puede maximizar la performance de la institución, "todo para la acreditación".

Sin embargo, la apropiación y rutinización en el empleo de técnicas pueden tener, paradójicamente, impactos negativos en la universidad al acrecentar aún más la burocracia y los mecanismos de control, junto con producir un mayor distanciamiento respecto del cuerpo académico. De este modo, anteponer la técnica por sobre lo sustantivo, si bien le otorga la ilusión de control y eficacia personal, también promueve el desplazamiento de las tareas de primer orden en las universidades. De esta forma, es una identidad profesional producida bajo las demandas de performatividad que posiciona a estos profesionales en terrenos en los cuales son requeridos para responder a las demandas de rendición de cuentas, pero también resistidos en las culturas organizacionales de las universidades.

\section{Discusión y conclusiones}

En el marco de los cambios económicos y sociales de las últimas décadas, las universidades han adoptado prácticas de trabajo asociadas a nuevos modelos de gestión provenientes de una lógica empresarial, con el fin de rendir cuentas y aumentar la transparencia. Lo anterior, sumado a la mayor permeabilidad de las instituciones a la evaluación externa —establecido por una ley de educación superior en Chile-, son factores que han promovido cambios organizacionales dentro de las universidades. Este es especialmente el caso del aseguramiento interno de la calidad, que se ha convertido en un nuevo ámbito de acción, que la normativa establece como una dimensión obligatoria y evaluable en las instituciones de educación superior. La gestión de la calidad articula aspectos académicos y administrativos y genera un nuevo territorio de desempeño profesional. Esta nueva área profesional, constituye un espacio de acción emergente caracterizado por la ambigüedad y falta de definición de sus funciones dentro de instituciones, con ámbitos altamente estructurados y estables en el tiempo. Además, debido a que no hay historia ocupacional previa, es esperable que la influencia externa permee más fuertemente a 
este grupo profesional, el cual puede ser moldeado por las nuevas y crecientes demandas de gestión universitarias.

Los profesionales son percibidos como "extraterrestres" al interior de las universidades, debido a la novedad e incomprensión de sus funciones, lo que se ve incrementado con la inminente aplicación de una ley que regula esta función, pero que no ha sido socializada ni debidamente acordada entre los actores. En este contexto, este artículo buscó contribuir a la comprensión de la construcción identitaria de los administradores de la calidad, dada la indefinición del campo que se ha abierto y del rol de estos nuevos actores. De acuerdo con sus relatos, los profesionales se perciben como pioneros en la instalación del aseguramiento de la calidad dentro de las universidades en las que se desempeñan. En este proceso, encontraron rechazos iniciales a sus acciones, por parte del cuerpo académico, debido a que representarían valores vinculados con la administración, los cuales son percibidos como contrarios a los fines de la academia. Estos resultados son consistentes con otras investigaciones. Por ejemplo, Feather (2015) plantea que las prácticas de gestión y mercantilización llevadas a cabo por los administradores académicos ha dificultado la relación con el cuerpo académico. Por su parte, Winter y O'Donohue (2012) encuentran que los académicos comparten una profunda antipatía hacia una ética dirigida por el mercado, la cual consideran que reduce la educación superior a una función económica. Por ello, la influencia interpersonal parece ser clave en la construcción del rol desempeñado, ya que les ha permitido ampliar las fronteras de su trabajo. Este despliegue de habilidades interpersonales, ha sido crucial para convencer a los académicos respecto de la importancia de sus funciones.

En consecuencia, se observa una tensión entre la necesidad de las universidades de contar con un cuerpo profesional capaz de responder a las demandas del entorno y una resistencia de los académicos a una lógica de burocratización. Las concepciones negativas hacia la gestión pueden dar cuenta de una ambivalencia, debido a que el personal docente está sobrecargado y requiere de apoyo para el trabajo administrativo, pero al mismo tiempo lo desprecia (Henkel, 
2000; 2007). De esta forma, "los académicos quieren gobernarse a sí mismos, no quieren dedicarse a la gestión y, sin embargo, niegan los derechos de gestión a los demás" (Dearlove, 1998, p. 73). En la medida en que el cuerpo académico no reconozca las contribuciones de los administradores académicos, los profesionales continuarán sintiéndose al margen de la corriente universitaria, intentando disminuir la ambigüedad de su rol y la ansiedad que ello les produce. La reducción de la incertidumbre se logra magnificando el valor de los aspectos formales, lo que a su vez converge y se retroalimenta de las demandas externas que, en Chile, tienen la particularidad de ser reguladas legalmente, por lo que su naturaleza está intrínsecamente recargada de requerimientos administrativos. En respuesta a ello, es esperable que aumente la burocratización de los procesos a cargo de quienes administren el aseguramiento de la calidad, con el fin de marcar un territorio y hacer visible su trabajo.

De acuerdo con el relato de los entrevistados en este estudio, se advierte un buen acoplamiento de ellos con la necesidad de dar respuestas a las agencias externas, mientras que el acoplamiento con la cultura académica y la tradición universitaria es más débil. Consecuentemente, al interior de las universidades se generan tensiones o espacios de juego de poder a través de micropolíticas que buscan favorecer una u otra orientación, dependiendo si se enfatiza la colegialidad o la burocratización.

Los resultados descritos muestran que su identidad profesional se está construyendo en el umbral, es decir, desde una posición caracterizada por "ni una cosa ni la otra"; es decir, no pertenecen a la planta de funcionarios administrativos que son "no-profesionales" (como estafetas o secretarias) ni al cuerpo académico. Esta dinámica contribuye a desvirtuar el sentido de la calidad que es el objeto de su función, alineando su trabajo fundamentalmente a la implementación de instrumentos y mecanismos, exigidos principalmente desde el mundo extraacadémico. Su mayor acoplamiento con el medio externo representado por las agencias de acreditación, las normas que regulan el funcionamiento universitario y la misma sociedad, les refuerza los significados instrumentales de la calidad. De este modo, al mismo tiempo que van fortaleciendo su identidad, se adaptan a 
las condiciones que establece el referente normativo, a través de una dinámica que busca el balance entre la concepción de sí mismos y la de quienes los rodean.

Por este motivo, se hace necesaria una mayor integración de las labores académicas y administrativas, porque es esperable que este estamento profesional continúe creciendo en las universidades. Como señala Whitchurch (2007), la superación de estas percepciones en las instituciones contemporáneas es una tarea clave para los líderes y directivos universitarios.

En conclusión, los administradores del aseguramiento de la calidad son profesionales que se integran al escenario de la educación superior, el cual se ha reconfigurando debido a la instalación de mecanismos de control y exigencias de rendición de cuentas públicas. Conforman un nuevo estamento de personal universitario que se especializa en la generación y validación de evidencias. Sin embargo, es posible plantear que los procesos de profesionalización de la gestión en el ámbito particular de la calidad, en muchos casos, han generado una distorsión del propósito original del sistema y del sentido de los procesos de aseguramiento de la calidad. Se ha suplantado la naturaleza primaria de las universidades, la que típicamente se distingue, entre otras cosas, por su orientación hacia el desarrollo del conocimiento y la contribución a la sociedad. Por ello, se vuelve fundamental articular el ámbito académico y el de gestión, con el fin de mantener en perspectiva los fines de la educación superior, al tiempo que puedan cumplirse las demandas de transparencia y cuentas públicas.

Construir una identidad profesional tiene que ver con la posibilidad de ser aceptado por otros desde lo que uno es y desde lo que uno propone. Si hay trabajo colaborativo se construye un espacio común en el que cada uno es visualizado como pieza relevante de un todo: es justamente este proceso el que parece estar todavía en curso en nuestra realidad. Si se logra visibilizar su rol y delinear un territorio de su incumbencia, sin tener que recurrir a la instrumentalización de los procesos de calidad, estos profesionales podrán consolidar un espacio y un rol que se articule con los modos que conforman el carácter e identidad académica y construir conjuntamente este nuevo 
tercer espacio, con un equilibrio entre la participación académica y administrativa.

\section{Referencias}

Allen-Collinson, J. (2006). Just 'non-academics'? Research administrators and contested occupational identity. Work, Employment \& Society, 20(2), 267-288. https://doi.org/10.1177/0950017006064114

Allen-Collinson, J. (2009). Negative 'marking'? University research administrators and the contestation of moral exclusion. Studies in Higher Education, 34(8), 941-954. https://doi. org/10.1080/03075070902755641

Anderson, G. (2006). Assuring quality/resisting quality assurance: Academics' responses to 'quality' in some Australian universities. Quality in Higher Education, 12(2), 161-173. https://doi. org/10.1080/13538320600916767

Ball, S. J. (2003). The teacher's soul and the terrors of performativity. Journal of Education Policy, 18(2), 215-228. https://doi.org/10.1080/0268093 022000043065

Barnetson, B. \& Cutright, M. (2000). Performance indicators as conceptual technologies. Higher Education, 40(3), 277-292.

Bernasconi, A. (2008). La profesionalización de la academia en Chile. Calidad en la Educación, 28, 15-27

Berríos, P. (2015). La profesión académica en Chile: crecimiento y profesionalización. En A. Bernasconi (Ed.), La educación superior de Chile: transformación, desarrollo y crisis (pp. 347-369). Santiago de Chile: Ediciones Universidad Católica.

Biggs, J. (2001). The reflective institution: Assuring and enhancing the quality of teaching and learning. Higher Education, 41(3), 221-238.

Braun, V. \& Clarke. V. (2006). Using thematic analysis in psychology. Qualitative Research in Psychology, 3(2), 77-101. https://doi. org/10.1191/1478088706qp063oa

Brown, A. D. \& Starkey, K. (2000). Organizational identity and learning: A psychodynamic perspective. Academy of Management Review, 25(1), 102-120. https://doi.org/10.5465/amr.2000.2791605

Brunner, J. J. (2017). La economía política del capitalismo académico: el caso de Chile. En H. Lavados y A. L. Durán (Eds.), Desafíos para el desarrollo universitario en Chile (pp. 159-231). Valencia: Tirant Humanidades. 
280 IDENTIDAD PROFESIONAL DE LOS ADMINISTRADORES DE LA CALIDAD EN UNIVERSIDADES CHILENAS: ENTRE LA INVISIBILIZACIÓN Y LA BUROCRATIZACIÓN - J. Scharager, P. Rodríguez

Brunner, J. J. y Pedraja-Rejas, D. (2017). Los desafíos de la gobernanza de la educación superior en Iberoamérica. Ingeniare. Revista Chilena de Ingeniería, 25(1), 2-7. https://doi.org/10.4067/s071833052017000100002

Brunner, J. J., Ganga-Contreras, F., y Rodríguez-Ponce, E. (2018). Gobernanza del capitalismo académico: aproximaciones desde Chile. Revista Venezolana de Gerencia, 23(1), 11-35.

Cáceres, C. (2007). Planeación estratégica en universidades del CRUCH. Revista Calidad en la Educación, 27, 108-150. https://doi.org/10.31619/ caledu.n27.221

Dearlove, J. (1998). The deadly dull issue of university "administration"? Good governance, managerialism and organising academic work. Higher Education Policy, 11(1), 59-79. https://doi.org/10.1057/palgrave. hep. 8380114

Deem, R., Hillyard, S., \& Ree D. M. (2007). Knowledge, higher education, and the new managerialism. The changing management of UK universities. New York: Oxford University Press.

Degn, L. (2015). Identity constructions and sensemaking in higher education -a case study of Danish higher education department heads. Studies in Higher Education, 40(7), 1179-1193. https://doi.org/10.1080/0307 5079.2014 .881345

Dubar, C. (1992). Formes identitaires et socialisation professionnelle. Revue Française de Sociologie, 33(4), 505-529. https://doi. org/10.2307/3322224

Consejo de Rectores de Universidades Chilenas, CRUCH. (2014). Anuario estadístico 2014. Recuperado de http://www.consejoderectores.cl/web/ pdf/anuario_2014/ANUARIO_2014_baja.pdf

Engebretsen, E., Heggen, K., \& Eilertsen, H. A. (2012). Accreditation and power: A discourse analysis of a new regime of governance in higher education. Scandinavian Journal of Educational Research, 56(4), 401417. https://doi.org/10.1080/00313831.2011.599419

Espinosa, A. B. (2015). Evaluación y acreditación de programas en México. Más allá de los juegos discursivos. Diálogos Sobre Educación, 3(2), 1-19. https://doi.org/10.1016/j.resu.2015.03.014

Feather, D. (2015). An alternative proposition to Lewis' views on the 'Constructions of professional identity in a dynamic higher education sector. Research in Post-Compulsory Education, 20(3), 324-343. https:// doi.org/10.1080/13596748.2015.1063300 
Fernández, E. (2009). Cambio de paradigma en la gestión de instituciones de educación superior. En A. Arata y E. Rodríguez (Eds.), Desafíos y perspectivas de la dirección estratégica de las instituciones universitarias (pp. 69-96). Santiago de Chile: Ediciones CNA-Chile.

Filippakou, O. (2011). The idea of quality in higher education: A conceptual approach. Discourse: Studies in the Cultural Politics of Education, 32(1), 15-28. https://doi.org/10.1080/01596306.2011.537068

Filippakou, O. (2017). The evolution of the quality agenda in higher education: The politics of legitimation. Journal of Educational Administration and History, 49(1), 37-52. https://doi.org/10.1080/00 220620.2017.1252738

Geoffroy Pitta, E. (2013). Estudio del sistema de aseguramiento de la calidad de la educación superior. Un análisis politológico de formulación de política pública. Revista Enfoques: Ciencia Política y Administración Pública, 11(19), 139-166.

González, E., Mora, A., Báez, M., Rodes, D., Ampuero, N., Ponce, ... Lagos. J. (2007). Análisis de la gestión institucional de la acreditación en universidades chilenas. En Acreditación y dirección estratégica para la calidad en las universidades (pp. 82-99). Santiago de Chile: Organización de las Naciones Unidas para la Educación, la Ciencia y la Cultura, Unesco-Centro Interuniversitario de Desarrollo, Cinda.

Harvey, L. (2004). The power of accreditation: Views of academics. Journal of Higher Education Policy and Management, 26(2), 207-223. https://doi. org/10.1080/1360080042000218267

Henkel, M. (2000). Academic identities and policy change in higher education. London: Jessica Kingsley Publishers.

Henkel, M. (2005). Academic identity and autonomy in a changing policy environment. Higher Education, 49, 155-176. https://doi.org/10.1007/ s10734-004-2919-1

Henkel, M. (2007). Can academic autonomy survive in the knowledge society? A perspective from Britain. Higher Education Research \& Development, 26(1), 87-99. https://doi.org/10.1080/07294360601166836

Lemaitre, M. J. (2015). Aseguramiento de la calidad: una política y sus circunstancias. En A. Bernasconi (Ed.), La educación superior de Chile: transformación, desarrollo y crisis (pp. 297-343). Santiago de Chile: Ediciones Universidad Católica.

Lewis, K. (2014). Constructions of professional identity in a dynamic higher education sector. Perspectives. Policy and Practice in Higher Education, 18(2), 43-50, https://doi.org/10.1080/13603108.2014.914107 
282 IDENTIDAD PROFESIONAL DE LOS ADMINISTRADORES DE LA CALIDAD EN UNIVERSIDADES CHILENAS: ENTRE LA INVISIBILIZACIÓN Y LA BUROCRATIZACIÓN - J. Scharager, P. Rodríguez

Morley, L. (2003). Quality and power in higher education. Maidenhead: SHRE, Open University McGraw-Hill Education, McGraw-Hill House.

McInnis, C. (1998). Academics and professional administrators in Australian universities: Dissolving boundaries and new tensions. Journal of Higher Education Policy and Management, 20(2), 161-173. https://doi. org/10.1080/1360080980200204

Newton, J. (2002). Views from below: Academics coping with quality. Quality in Higher Education, 8(1), 39-61. https://doi. org/10.1080/13538320220127434

Parri, J. (2006). Quality in higher education. Vadyba Management, 2(11), 107-111.

Rivera, F., Astudillo, P., y Fernández, E. (2009). Información y toma de decisiones: oficinas de análisis institucional en universidades chilenas. Revista Calidad en la Educación, 30, 161-178. https://doi.org/10.31619/ caledu.n30.176

Scharager, J. (2018). Quality in higher education: The view of quality assurance managers in Chile. Quality in Higher Education, 24(2), 102116. https://doi.org/10.1080/13538322.2018.1488395

Scharager, J. (2017). Nuevos actores en un viejo escenario: la profesionalización de la gestión de la calidad académica en Chile, 1990-2015 (Tesis doctoral). Universidad de Leiden, Ámsterdam, Holanda.

Servicio de Información de la Educación Superior, SIES. (2010). Evolución de la matrícula de educación Superior en Chile. Periodo 1990-2009. Santiago de Chile: Autor.

Servicio de Información de la Educación Superior, SIES. (2014). Panorama de la educación superior en Chile 2014. Santiago de Chile: Autor.

Seyd, R. (2000). Breaking down barriers: The administrator and the academic. Perspectives: Policy and Practice in Higher Education, 4(2), 35-37. https:// doi.org/10.1080/136031000412892

Szekeres, J. (2004). The invisible workers. Journal of Higher Education Policy and Management, 26(1), 7-22. https://doi.org/10.1080/13600800420 00182500

Szekeres, J. (2011). Professional staff carve out a new space. Journal of Higher Education Policy and Management, 33(6), 679-691. https://doi.org/10. 1080/1360080X.2011.621193

Thomas, R. \& Davies, A (2002). Gender and new public management: Reconstituting academic Subjectivities. Gender, Work \& Organization, 9(4), 372-397. https://doi.org/10.1111/1468-0432.00165 
Van Gennep, A. (2008). Los ritos de paso. Madrid: Alianza.

Wastell, D. G. (1996). The fetish of technique: methodology as a social defense. Information systems journal, 6(1), 25-40. https://doi. org/10.1046/j.1365-2575.1996.00104.x

Whitchurch, C. (2006). Who do they think they are? The changing identities of professional administrators and managers in UK higher education. Journal of Higher Education Policy and Management, 28(2), 159-171. https://doi.org/10.1080/13600800600751002

Whitchurch, C. (2007). The changing roles and identities of professional managers in UK higher education. Perspectives, 11(2), 53-60. https:// doi.org/10.1080/13603100701259022

Whitchurch, C. (2008). Shifting identities, blurring boundaries: The changing roles of professional managers in higher education. Recuperado de http:// escholarship.org/uc/item/3xk701cn

Whitchurch, C. (2013). Reconstructing identities in higher education: The rise of third space professionals. London: Routledge.

Watermeyer, R. (2015). Lost in the 'third space': The impact of public engagement in higher education on academic identity, research practice and career progression. European Journal of Higher Education, 5(3), 331-347. https://doi.org/10.1080/21568235.2015.1044546

Winter, R. (2009). Academic manager or managed academic? Academic identity schisms in higher education. Journal of Higher Education Policy and Management, 31(2), 121-131. https://doi. org/10.1080/13600800902825835

Winter, R. \& O'Donohue, W. (2012). Academic identity tensions in the public university: Which values really matter? Journal of Higher Education Policy and Management, 34(6), 565-573. https://doi.org/10.1080/136 0080X.2012.716005

Ylijoki, O. H. (2005). Academic nostalgia: A narrative approach to academic work. Human Relations, 58(5), 555-576. https://doi. org/10.1177/0018726705055963

Recibido: 16/10/2018

Aceptado: 27/05/2019 